\title{
XXVII. On the nebular hypothesis.-V. Æthereal nodes
}

\section{Pliny Earle Chase LL.D. S.P.A.S.}

To cite this article: Pliny Earle Chase LL.D. S.P.A.S. (1877) XXVII. On the nebular hypothesis. - V. Æthereal nodes, Philosophical Magazine Series 5, 3:17, 203-211, DOI: $10.1080 / 14786447708639219$

To link to this article: http://dx.doi.org/10.1080/14786447708639219

曲 Published online: 13 May 2009.

Submit your article to this journal $[\pi$

Џ Article views: 2

Q View related articles $\asymp$ 
ing. The magnetic moment, then, was sensibly zero. I then slid the coil along its railway, and, by aid of a small needle very strongly hardened, and rendered sensibly astatic by a compensating bar, ascertained the existence of energetic opposite polarities at the very extremity of the bar and a little nearer its middle. This delicate but absolutely conclusive experiment, thanks to the regularity and symmetry of the results obtained, cannot leave any doubt of the legitimacy of the induction which guided us in this portion of our investigation.

In brief, these last experiments seem to make it evident that there exists between the temporary and the permanent magnetization a sort of independence more decided than had hitherto been admitted. I persist in thinking that it must be attributed, at least in part, to a difference of origin, resulting from a real magnetic heterogeneity in the steel *

XXVII. On the Nebular Hypothesis.-V. Athereal Nodes. By Puint Earle Chase, LL.D., S.P.A.S., Professor of Philosophy in Haverford College.

[Continued from vol, ii. p. 202.]

QNE of the most important corollaries of the theory of universal gravitation is tersely stated by Stockwell $\uparrow$ as follows:- "The amount by which the elements of any planet may ultimately deviate from their mean values can only be determined by the simultaneous integration of the differential equations of those elements, which is equivalent to the summation of all the infinitesimal variations arising from the disturbing forces of all the planets of the system during the lapse of an infinite period of time." Therefore, within the limits of secular eccentricity, the result is the same as if the nebular hypothesis were true.

There should, then, be tendencies, in the neighbourhood of every inert particle which floats in an elastic medium, to the formation of harmonic nodes of various kinds; and the sum of such tendencies should fix loci of cosmical aggregation before there had been any considerable shapings of definite mass. The subsequent values of relative mass would depend upon mutual conditions of equilibrium between various forms of living force. But such accordances as would be thus produced, however interesting and however striking they might be deemed, would furnish no more conclusive evidence of the nebular theory, as popularly interpreted, than of the Cartesian

* See Annales de l'Ecole Normale Supérieure, t. iii. p. 52.

† Smithsonian Contributions, 232. viii. 
vortices. All assumptions as to the nature of ultimate physical force are now, and perhaps always will be, mere assumptions. Still, like geometrical diagrams, they may help to fix the mind upon ultimate physical resultants, and thus serve a useful purpose.

The velocity of rotation varies as the square root of the velocity of gravitating fall. Therefore, if the velocity of planetary revolution $(\sqrt{g r})$ at Sun's equatorial surface be taken to represent the velocity of athereal rotation at the same point, the present æthereal atmospheric limit, at which the equatorial velocity of rotation would be equal to that of the æthereal undulations which drive particles towards centres of inertia, is near the outer limit of the asteroidal belt (at $\sqrt{ }$ light-modulus $=688.3$ solar radii $=3.204$ mean vector-radii of Earth). The mean proportional between Earth's mean distance and Saturn's secular aphelion is 32216 .

We have found ${ }^{*}$ that the velocity at the methereal atmospheric limit (the velocity of light) is the limiting radial velocity at the point of equilibrium between the velocity of complete dissociation and the velocity of incipient aggregation. The limiting tangential velocity, at the point of equilibrium between complete aggregation and incipient dissociation, is therefore $\dagger \frac{1}{\pi}$ of the velocity of light, or the velocity of æthereal rotation at 219.09 solar radii, Earth's mean distance being $214 \cdot 86$.

We have also found $\ddagger$ that Jupiter, the largest extra-asteroidal, and Earth, the largest intra-asteroidal planet, are connected by a common limiting radial velocity, the indications pointing to nucleal or rotating influences in the casse of Earth, and to atmospheric or orbital influences in the case of Jupiter. Circular-orbital velocity varios as $g^{\frac{1}{4}}$. The relations of $g$ to $\sqrt{\mathrm{M}}(1)$, and of Earth's position to the unit of solar aggregation (2), lend importance to the approximate equality between $\log (\sqrt{\mathrm{M}})^{\frac{1}{4}}=\cdot 709444$ and $\log \mathrm{rad}$. . ec. $(4 \div \oplus)=\cdot 716237$. (3)

Alexander showed $\S$ that Jupiter and Saturn are so related as to suggest a possible early mutual participation in a common nucleal vis viva, as if they had been formed, by interior and exterior condensation, from the same nebulous belt. I have shown $\|$ that the atmospheric radius varies as the $\frac{4}{3}$ power of

* "Correlations of Central Force," Phil. Mag. September 1876, $\eta$, ı.

+ Ibid. $\zeta$. $\ddagger$ Ibid. $\lambda$.

\$ Smithsonian Contributions, 280, p. 38.

II Correlations, $k, \nu$. The present value of modulus in solar radii is given in $\nu$. 
the nucleal radius. Therefore if $\mathrm{M}^{\frac{1}{d}}$ represents Jupiter's position (3) at the extremity of a nucleal radius, the corresponding atmospheric radius $=(\sqrt[8]{\bar{M}})^{\frac{4}{3}}=\mathbf{M}^{\frac{1}{6}}$, of which the logarithm is .945926 . The logarithm of $h$ secular perihelion $\div \oplus$ mean radius vector is $\cdot 941236$.

The secular perihelion of Venus is near the æthereal nucleal limit (1), (4). $\log (\sqrt{\mathrm{M}})^{\frac{3}{4}}=2 \cdot 128332 ; \log$ o secular perihelion $=2 \cdot 159680 ; 2 \cdot 159680-2 \cdot 128332=\cdot 031348=$ $\log 1.0748$; q secular aphelion $\div$ mean radius vector $=$ 1.0763 .

These approximations point to æthereal influences on the principal planets, both in the supra- and in the infra-asteroidal belt, and to early special nucleal condensation in the inner belt. The latter indication is strengthened by the greater density of the interior planets, by the many harmonic relations which are based on Earth's distance as a primitive unit, and by Earth's position near the centre of the infra-asteroidal belt. Mercury's secular peribelion (-29743) + Mars secular aphelion $(1 \cdot 73633)=2 \cdot 03376 ; 2 \cdot 03376 \div 2=1 \cdot 01688 ;$ Earth's present aphelion $=1 \cdot 01678$.

The nucleo-atmospherie relations of Earth and Jupiter (3) are further shown by the fact that a nucleal expansion of Sun to Farth's secular perihelion would involve an atmospheric expansion to $\left(200 \cdot 307^{\frac{4}{3}}=\right) 1172$ solar radii. Jupiter's mean aphelion $=1166 \cdot 61$ solar radii

The present light-modulus $(\log \mathrm{M} \div \odot r=5 \cdot 675554)$ : Earth's accelerative radius $\left(\log 2 \rho^{2}=4 \cdot 965340\right)::$ Jupiter's secular aphelion $(\log =741881)$ : Earth's secular aphelion $(\log =$ -028463).

Earth's rotating, relatively to its orbital, velocity has been accelerated $366 \cdot 256$ times since its theoretical nebular rupture. This represents the ratio of Earth's nucleal rupturing- to Sun's aggregating-radius (2). For if we let $\rho=$ superficial radius and velocity of perfect fluidity in the rthereal nucleus (1), $\rho \sqrt{ } 2=$ radius of dissociating velocity, and $2 \rho^{2}=$ radius of rupturing vis viva; $\log \rho(=\bigoplus$ secular perihelion $)=2 \cdot 301695$, $\log \left(2 \rho^{2} \div 219 \cdot 0894\right)=2 \cdot 563791=\log 366 \cdot 253$.

The increased acceleration of Jupiter's angular velocity, relatively to its nucleal companion Earth, is such as would be due to the difference of orbital velocities at the outer and inner edges of the Jovi-Telluric belt. Log 4 secular aphelion $(\cdot 741881)-\log \oplus$ secular perihelion $(\overline{1} \cdot 969540)=\log 2 \cdot 433^{2}$. 24 h. $\div 2 \cdot 433=9$ h. 51 m. 49 s. . . . . . . . . (10) 
Jupiter's rotating, relatively to its orbital, velocity has been accelerated in the ratio of its mean rupturing- to Sun's aggregating-radius. For $\log \rho(=4$ mean perihelion $)=3.029231$; $\log \left(2 \rho^{2} \div 219 \cdot 0894\right)=4 \cdot 018865=\log 10443 \cdot 97 ; 4332 \cdot 585 \mathrm{~d}$. $\div 10443.97=9$ h. $57 \mathrm{~m} .22$ s. See (7), (35), (48). . (11)

Saturn's rotating, relatively to Jupiter's orbital, velocity has been accelerated in the ratio of Jupiter's nucleal rupturing- to Sun's aggregating-radius. For $\log \rho(=4$ sec. per. $)=3.021137$; $\log \left(2 \rho^{2} \div 219 \cdot 0894\right)=4 \cdot 022677=\log 10061 \cdot 83 ; 4332.585 \mathrm{~d}$. $\div 10061 \cdot 83=10$ h. 20 m. 3 s.

Saturn's rotating, relatively to its orbital, velocity has been accelerated in the ratio of Saturn's initial rupturing-radius to Earth's radius of rupture. For $\log \rho(=h$ sec. aph. $)=3 \cdot 346812$; $\log \left(2 \rho^{2} \div 200 \cdot 307\right)=4 \cdot 391929=\log 24656 \cdot 36 ; 10759 \cdot 22 \mathrm{~d}$. $\div 24656 \cdot 36=10$ h. 14 m. $4 \mathrm{~s}$.

The rotating velocity of Mars, relatively to its orbital velocity, has been accelerated nearly in the ratio of its nucleal rupturing-radius to Earth's secular aphelion. For $\log \rho(=\delta$ sec. per. $)=2 \cdot 449775 ; \log \left(2 \rho^{2} \div 229 \cdot 413\right)=2 \cdot 839962=\log$ $691 \cdot 77 ; 686.98$ d. $\div 691 \cdot 77=23$ h. 49 m. 49 s. . . (14)

The rotating velocity of Venus, relatively to its orbital velocity, has been accelerated in the ratio of its mean rupturingradius to Earth's mean perihelion. For $\log \rho=2 \cdot 191493$; $\log \left(2 \rho^{2} \div 207 \cdot 583\right)=2 \cdot 366824=\log 232 \cdot 715 ; 224 \cdot 7$ d. $\div$ $232 \cdot 715=23 \mathrm{~h} .13 \mathrm{~m} .36 \mathrm{~s}$.

The rotating velocity of Mercury, relatively to its orbital velocity, has been accelerated in the ratio of its initial rupturingradius to Sun's aggregating-radius. For $\log (\rho=\varnothing$ sec. aph.) $=1.990608 ; \log \left(2 \rho^{2} \div 219.0894\right)=1.941619=\log 87.422 ;$ 87.97 d. $\div 87 \cdot 422=24$ h. 9 m. 2 s. . . . . . . . (16)

Jupiter's secular aphelion $(5 \cdot 5193)$ is a mean proportional between Earth's mean distance and Neptune's secular aphelion $(30 \cdot 4696)$. See $(27)$ to $(29)$

The secular perihelion of Uranus $(17 \cdot 688)$ is at the centre of the supra-asteroidal belt. For Neptune's secular aphelion $(30 \cdot 47)+$ Jupiter's secular perihelion $(4 \cdot 886)=35 \cdot 356$; $35 \cdot 356 \div 2=17 \cdot 678$. . . . . . . . . . (18)

The secular perihelion of Uranus, or its locus of nebular rupture, is also a mean proportional between Saturn's secular aphelion $(10 \cdot 343)$ and Neptune's mean aphelion $(30 \cdot 336)$.

The centres of the outer and inner planetary belts are so related that the mean distance of Uranus $(19 \cdot 184)$ and Earth's rupturing-locus (sec. per. $=\cdot 932$ ) are at apsides of a major axis 
which would be traversed by light-undulations in the time of planetary revolution at Sun's surface. For $19 \cdot 184+\cdot 932=$ $20 \cdot 116 ; 688 \cdot 3 \times 2 \pi \div 214 \cdot 86=20 \cdot 128$. . . . . . (20)

The major axis of the November meteoric orbit is also nearly equivalent to the major axis of these primeval light-undulations. For the meteoric period $=33 \cdot 25$ yrs. ; $2 \times 33 \cdot 25^{\frac{2}{3}}=$ 2068 . . . . . . . . . . . . . . (21)

When Sun's surface of dissociation was at the extremity of Earth's mean radius vector, the locus of complete aggregation, or the vertex of the stellar-solar paraboloid*, was at Mercury's present perihelion $(\cdot 3187)$. For $1 \div \pi=\cdot 3184$.

The orbital velocity varies as the $\frac{1}{4}$ power of the gravitating velocity (3). The orbital velocity at the mean aphelion of the intra-asteroidal belt is equivalent to the mean velocity of the centripetal gravitating impulses beyond the belt. For log (sec. aph. $\psi \times$ sec. aph. $\delta)^{\frac{1}{8}}=\cdot 215437 ; \log$ mean aph. $\delta=$ $\cdot 215944$.

The mean velocity of the centripetal gravitating impulses in the principal nucleal belt is also equivalent to the meanaphelion intra-asteroidal velocity. For $\log$ (sec. aph." h $x$ mean $\psi)^{\frac{1}{8}}=\cdot 216362$.

There is therefore an equivalence between the mean exterior and the mean nucleal gravitating impulses beyond the Telluric belt. For $\log \left(\text { sec. per. } \Psi \times \text { sec. aph. } \delta^{*}\right)^{\frac{3}{2}}=.855866 ; \log ($ sec. aph. $h \times$ mean per. 4$)^{\frac{1}{2}}=855450$. . . . . . . . (25)

The orbital velocity varies as the $\frac{1}{2}$ power of the rotating velocity of a varying nebula. The mean orbital velocity due to nebular action in the Neptuno-Uranian belt is equivalent to the rotating velocity at the locus of nebular rupture in the principal nucleal belt. For $\log (\text { mean per. } \Psi \times \text { mean } \$)^{\frac{1}{4}}=$ $\cdot 689039$; log sec. per. $4=\cdot 688982$.

The initial rupturing-position of the centre of planetary mass (17) is determined by the mean influence of the intraasteroidal centre (6), the supra-asteroidal centre (18), and the nebular centre of planetary inertia. For $\log ($ mean $\oplus \times$ sec. per. $\delta \times$ mean $\mathfrak{h})^{\frac{1}{3}}=\cdot 742338$; log sec. per $4=\cdot 741881$.

The same position is also a mean proportional between the centre of the supra-asteroidal and the outer limit of the intra-

* It was inadvertently stated in the article on "Correlations," that there are nine abscissi $s$ between Neptune and $\alpha$ Centauri. There are nine in my original paraboloid; but if the vertex is taken at the locus of complete solar aggregation, there are eighteen. 
asteroidal belt. For $\log$ (sec. per. $\widehat{o} \times$ sec. aph. $\left.\delta^{1}\right)^{\frac{1}{2}}=$ $\cdot 743575$.

The nebula-rupturing position of the centre of planetary mass is at the centre of the initial planetary system. For sec. aph. $\Psi(30.470)$ - sec. aph. $6(20.679)=2 \times$ sec. per. 4 $(4 \cdot 886)$.

The initial position of mean planetary inertia is determined by the mean positions of the rupturing-loci of the two principal two-planet belts. For $\log (\hat{\circ} \times 4)^{\frac{1}{2}}=\cdot 999583$; log mean aph. $h=1 \cdot 000003$.

The atmospheric limit (4) of the infra-asteroidal belt is determined by positions of Sun, Jupiter, and Neptune. For $\log \left(\overline{4 \times \psi^{\frac{1}{2}}} \div \odot r\right)=3.429079$; $\log (\text { sec. aph. } \delta \div \odot r)^{\frac{4}{3}}=$ $3 \cdot 429048$.

The atmospheric limit of the initial position of the infraasteroidal centre is determined by positions of Sun, Jupiter, and Saturn. For log (sec. per. $4 \times$ sec. per. $\left.h^{\frac{1}{2}} \div \odot r\right)=$ $3 \cdot 147264 ; \log$ (sec. aph. $\oplus \div \odot r)^{\frac{4}{8}}=3 \cdot 147491$. . . . (32)

The atmospheric limit of the initial tendency to infraasteroidal rupture is determined by positions of Sun, Jupiter, and Earth. For log (mean per. $4 \times \oplus)^{\frac{1}{2}}=2 \cdot 680693$; $\log$ (sec. aph. $\Varangle \div \odot r)^{\frac{4}{3}}=2 \cdot 680615$. . . . . . . . . . (33)

The atmospheric limit at the inner locus of infra-asteroidal rupture is the nucleal rupturing-limit of Mars relatively to Earth. For $\log$ (sec. per. $\Varangle \div \odot r)^{\frac{4}{3}}=2 \cdot 420721=\log 1.226 \oplus$ r. vec.; (sec. per. $\delta \div \oplus)^{\frac{3}{4}}=1 \cdot 225$. . . . . . . . (34)

The atmospheric limit at the central locus of infra-asteroidal rupture is at Jupiter's mean aphelion. For log (sec. per. $\oplus \div \odot r)^{\frac{4}{3}}=3.068927 ; \log ($ mean aph. $4 \div \odot r)=3.066743$. (35)

The atmospheric limit at the rupturing-locus of Mars is near the rupturing-limit of Saturn. For $\log$ (sec. per. $\delta \div \odot r)^{\frac{4}{3}}$ $=3.266367$; log sec. per. $h \div \odot r=3 \cdot 273391 ; 3 \cdot 273391-$ $3 \cdot 266367=007024=\log 1 \cdot 0163$. This indicates a similarity of contraction at the centre (6) and at the outer limit of the belt.

The atmospheric limits of the Venus belt, as determined by reference to the rupturing-position of Mercury, are in or near the Earth belt. For $\log (q \div \text { see. per. } \nsucceq)^{\frac{4}{3}} \div \oplus$ r. vec. $=$ $\overline{1} \cdot 942238 @ 024175 ; \log \oplus=\overline{1} \cdot 969540 @ \cdot 028463$.

The atmospheric limits of the Earth belt, referred to the rup- 
turing-position of Mercury, are within the Mars belt. For log $(\oplus \div \text { sec. per. } \wp)^{\frac{4}{3}}=\cdot 131591 @ \cdot 210155 ; \log \delta=\cdot 117620 @$ $\cdot 239631$.

The atmospheric limits of the Mars belt, referred to the rupturing-position of Mercury, are within the asteroidal belt. (39)

The atmospheric limit at Venus's mean aphelion, referred to Mercury's mean locus, is at Earth's rupturing-locus. For $\log (\text { mean aph. } q \div \Varangle)^{\frac{4}{3}}=\cdot 382120 ; \log ($ sec. per. $\oplus \div \xi)=$ .381719 .

The atmospheric limit at Earth's initial locus, referred to the initial locus of Mercury, is at the mean perihelion of Mars. For $\log (\text { sec. aph. } \oplus \div \text { sec. aph. } \Varangle)^{\frac{4}{3}}=466876$; log (mean per. $\delta \div$ sec. aph. $\Varangle)=\cdot 468819$.

The initial locus of Earth is at the mean aphelion thermal radius of Venus. For $\log 1.4232$ (mean aph. $q \div \oplus$ ) $=\cdot 027677$; $\log$ sec. aph. $\oplus=028463$. . . . . . . . . . (42)

The atmospheric limit at the ruptaring-locus of Mars (36), referred to the rupturing-locus of Venus, is near the mean aphelion of Mars. For log (sec. per. $\delta \div$ sec. per. $q)^{\frac{4}{3}}=\cdot 214318$; $\log$ mean aph. $\delta=\cdot 215944$.

The inner atmospheric limit of the Jupiter belt, referred to the rupturing-locus of Venus, is at Saturn's mean distance. For $\log$ (sec. per. $4 \div$ sec. per. +$)^{\frac{4}{3}}=976134 ; \log \hbar=$ .979496 .

The outer atmospheric or initial limit of the Jupiter belt, referred to the rupturing-locus of Venus, is near Saturn's initial locus. For $\log$ (sec. aph. $4 \div$ sec. per. $q)^{\frac{4}{3}}=1 \cdot 046666$; log sec. aph. $h_{2}=1 \cdot 014657 ; 1 \cdot 046666-1 \cdot 014657=\cdot 032009$ $=\log 1 \cdot 077=\log ($ sec. aph. $\div$ mean 6$)$. See (3) to $(5)$, (20).

The inner atmospheric limit of the Saturn belt, referred to the rupturing-locus of Venus, is at the initial locus of Uranus. For $\log$ (sec. per. $h \div$ sec. per. $q)^{\frac{4}{3}}=1.312473$; log sec. aph. $\diamond=1 \cdot 315531$.

The outer atmospheric limit of the Saturn belt, referred to the rupturing-locus of Mars, is also at the initial locus of Uranus. For $\log$ (sec. aph. $h \div$ sec. per. $\left.{ }^{*}\right)^{\frac{4}{3}}=1 \cdot 313669$. (47)

The inner atmospheric limit of the Jupiter belt, referred to Earth's rupturing-locus, is near Saturn's rupturing-locus. For log sec. per. $4 \div$ sec. per. $\oplus)^{\frac{4}{3}}=928796$; log. sec. per. $h=$ $.941236 ; \cdot 941236-.928796=\cdot 012440=\log 1 \cdot 029$.

Phil. Mag. S. 5. Vol. 3. No. 17. March 1877. 
The outer atmospheric limit of the Jupiter belt, referred to Earth's rupturing-locus, is at the mean aphelion of Saturn. For $\log$ (sec. aph. $4 \div$ sec. per. $\oplus)^{\frac{4}{3}}=.999328 ; \log$ mean aph. $h=1.000003$.

The mean.atmospheric limit of the Saturn belt, referred to Earth's mean locus, is near the mean aphelion of Uranus. For $\log (h \div \oplus)^{\frac{4}{3}}=1 \cdot 305995$; log mean aph. $\AA=1 \cdot 301989$. (50)

The atmospheric limit at Jupiter's mean aphelion, referred to the rupturing-locus of Mars, is at Saturn's rupturing-locus. For $\log \left(\text { mean aph. } 4 \div \text { sec. per. } \delta^{7}\right)^{\frac{4}{3}}=.940244$; log sec. per. $h=.941236$.

The mean atmospheric limit of the Uranus belt, referred to Jupiter's rupturing-locus, is at Neptune's mean aphelion. For $\log (\$ \div \text { sec. per. } 4)^{\frac{4}{3}}=1 \cdot 480913$; log mean aph. $\Psi=$ $1 \cdot 481951$.

The same limit (52), referred to Jupiter's mean perihelion, is at Neptune's mean locus. For $\log (\hat{0} \div \text { mean per. } 4)^{\frac{4}{3}}=$ $1 \cdot 478215 ; \log \Psi=1 \cdot 477611$. . . . . . . . . (53)

The same limit, referred to Jupiter's mean position, is at Neptune's rupturing-locus. For $\log \left(\frac{1}{5} \div 4\right)^{\frac{4}{3}}=1 \cdot 471828$; log sec. per. $\psi=1.471268$. . . . . . . . . . (54)

The important influence of Earth's position at a centre of early nucleal condensation is also shown by the simplicity of relations between Earth's radius vector and the secular epicyclical undulations of the supra-asteroidal planets.

Earth and Sun are convertible points of suspension for a linear pendulum equivalent to the secular excursion of Uranus. For $3 \div 38 \cdot 365=0782$; the maximum eccentricity of Uranus is 0780 . See $(20),(21)$. . . . . . . . . . (55)

The excursion of Saturn is nearly equivalent to the atmospheric limit of a nucleas which has Earth's thermal radius $\left(1 \cdot 4232^{\frac{4}{3}}=1 \cdot 601\right)$. For $1 \cdot 601 \div 19 \cdot 078=\cdot 0839 ;$ the maximum eccentricity of Saturn is $\cdot 0843$.

The excursion of Jupiter is equivalent to the mean radius of rotating inertia at Earth's orbit $(\sqrt{\cdot 4}=\cdot 6325)$. For $\cdot 6325$ $\div 10 \cdot 406=06078$, Jupiter's maximum ecentricity being $\cdot 06083$.

The excursion of Neptune is in the inverse ratio of its own coefficient $\left(\frac{8}{7}\right)$, and in the direct ratio of the coefficient of Uranus $\left(\frac{7}{8}\right)$, in the abscissas of the solar aggregating-paraboloid. For $\frac{7}{8} \div 60 \cdot 074=\cdot 0146$; Neptune's maximum eccentricity is 0145 . 
The following Table shows the closeness of approximation, (theoretical - observed) $\div$ observed in each of the foregoing comparisons:-

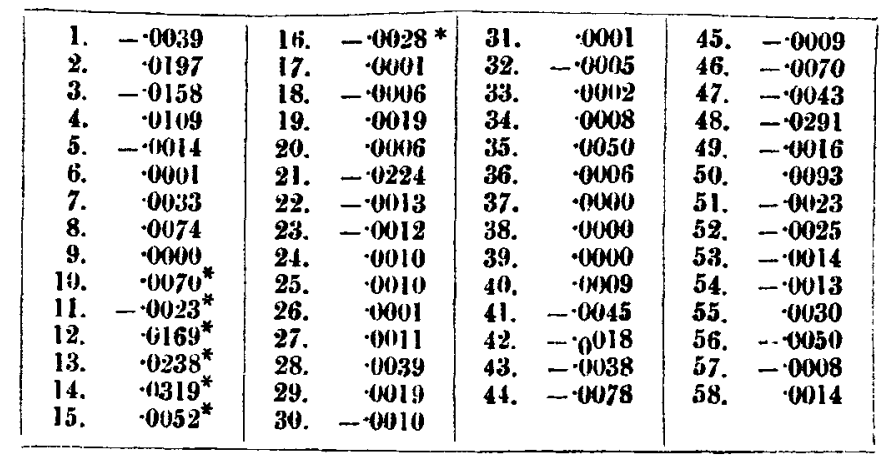

XXVIII. On the Speed of Signalling through Heterogeneous Telegraph Cireuits. By Oliver Heaviside†.

WHEN the first trials of speed of working were made on the Anglo-Danish cable, then recently laid (September 1868), it was found that a considerably higher speed could be reached in one direction than in the other. The "line "portion of the circuit consisted of a land-line on the English side of 240 ohms resistance, then a cable of $2500 \mathrm{ohms}$ resistance and capacity 120 microfarads, and a land-line on the Danish side of 1250 ohms-all approximate. The circuit was completed through a battery of $150 \mathrm{ohms}$ at one end and a Wheatstone's receiver of $750 \mathrm{ohms}$ at the other, the circuit being worked on the earth-to-earth principle, i. e. without condensers. But although the battery and receiver at each end were the same, or nearly so, the maximum speed obtained withWheatstone's transmitter, making mechanically exact signals, was 40 per cent. higher from England to Denmark than from Denmark to Englandf. This unexpected result was abundantly contirmed by the subsequent experience of every-day practice, which proved the existence of a difference in working-speed in opposite directions varying from 20 to 40 per cent. at different times, mainly according to the state of insulation of the land-lines.

* According to Herschel.

+ Communicated by the Author.

I It may be interesting to state the actual speeds obtained on this circuit with different instruments. Morse, 60 to 75 letters per minute; Wheatstone's transmitter and receiver, 90 to 140 letters per minute; Wheatstone's transmitter and Thomson's recorder, 300 to 360 letters per minute : in all cases without condensers. 